\title{
Mounting and support for pseudo biaxial Scheimpflug focusing for unity-magnification, high-speed particle velocimetry
}

\author{
S. L. Ribergård ${ }^{1 *}$, P. J. Olesen ${ }^{1}$, N. S. Jensen ${ }^{1}$, J. S. Nielsen ${ }^{1}$, C. M. Velte ${ }^{1}$ \\ ${ }^{1}$ Turbulence Research Laboratory, Technical University of Denmark, Department of Mechanical \\ Engineering, Kgs. Lyngby, Denmark \\ *silari@mek.dtu.dk
}

\begin{abstract}
A camera mount that can support both heavy cameras and heavy optics allowing a total of seven degrees of freedom shared between them has been designed. This allows for Scheimpflug focusing along one or two axes. A paper proposing a solution to two-axes Scheimpflug focusing has been examined and a new nomer is proposed for two-axes Scheimpflug focusing. The newly designed mounts allow for a broader range of solutions for combinations of positioning and alignment than traditional Scheimpflug mounts.
\end{abstract}

\section{Introduction}

Scheimpflug focusing has been widely applied in both scientific research and specialized photography when the desired object plane and focus plane do not coincide. This is particularly of interest when making experiments using particle image velocimetry with multiple cameras or single cameras with difficult optical access. Stereoscopic and tomographic methods present challenges, as described in classical PIV-textbooks, (Raffel et al., 2018) and Adrian and Westerweel (2011), for which several commercial solutions have been made, which allows fulfillment of the Scheimpflug criterion ensuring alignment of the Image Plane and the Object Plane. These solutions are all designed to sit between the camera and the lens, letting either the camera carry the weight of the adapter and lens, like the LaVision Manual Scheimpflug Mount, or letting the adapter carry both, like the PIVTECH Single-Axis Scheimpflug Camera Adapter. This is fine when working with light-weight equipment or just a heavy camera, however, when attempting high-speed unitymagnification measurements both sides of the mount tends to increase in bulk and weight. For the setup being established at the Turbulence Research Laboratory at DTU Mechanical Engineering in Denmark, a solution was needed that could carry the heavy Vision Research Phantom v2640 along with the lens setup consisting of the AF-S Nikkor 300mm f/4D IF-ED with a AF-S Teleconverter TC-20E III, all the while allowing for fulfillment of the Scheimpflug criterion. The outcome is the design presented herein. A camera mount that, through its seven degrees of freedom, can reach complicated solutions for the Scheimpflug criterion while carrying the heavy equipment needed for our desired experiments.

\section{Pseudo biaxial Scheimpflug focusing}

Already at the start of the previous century, Theodore Scheimpflug patented a method for what would become known as Scheimpflug focusing in Scheimpflug (1904). In this, he presents both the mathematical and intuitive backgrounds for achieving a focus coinciding with an object plane not parallel to the image plane of the camera. This method can be considered a single-axis, or monoaxial, Scheimpflug focusing, as it only considers a single axis of rotation. In many cases, this is sufficient, assuming the ability to align the equipment such that the normals of the three planes (image, object and lens) are coplanar. This, however, is not always the most feasible approach, so in 2001, Stephen Walker published a paper in which he discuss biaxial Scheimpflug focusing, Walker (2001). He derives a mathematical solution resulting in a slanted Scheimpflug line, i.e. the common line of the three planes. In our group, this approach was investigated, 
and while we came to similar results, we could not help feeling that this seemed artificial. As it turns out, we're now convinced that there are no such thing as true biaxial Scheimpflug focusing, only what we have dubbed "pseudo biaxial" Scheimpflug focusing. As shown by Euler, any one rotation can be decomposed into two or more rotations along different axes or vice versa. Thus, what Walker presented is "simply" a monoaxial focusing decomposed into two separate rotations. This, however, does indeed have its place, as rotating around the optical axis of the camera might not always be possible. For this reason, we found the nomer "pseudo biaxial" captures the principle better than "two-axes". To achieve focus across the sensor, the same principle as for single-axis Scheimpflug focusing still applies, understood as that the normals of the three planes must be coplanar.

\section{Design}

Initial experimental designs complicated the camera setup, forcing the decision to design the camera mounts for flexibility. To accommodate the need for splitting up the rotation needed to satisfy the Scheimpflug condition into two separate rotations, the mount needs to allow for rotations and translations of both camera and lens, independently. This solution can rotate the entire mount with both camera and lens at once, translate the camera along its optical axis and both rotate and translate the lens independently from the camera. The final design has a total of seven degrees of freedom, as seen in Table 1 . In Figure 1 a kinematic sketch of the design principle can be seen, showing the translations and rotations relative to each other.

Table 1: List of degrees of freedom (DoF).

\begin{tabular}{l|l} 
DoF & Description \\
\hline R1 & Pitch of entire mount \\
R2 & Pitch of lens \\
R3 & Yaw of entire mount \\
R4 & Yaw of lens \\
T1 & Axial translation of camera \\
T2 & Vertical translation of lens \\
T3 & Horizontal traverse translation of lens
\end{tabular}
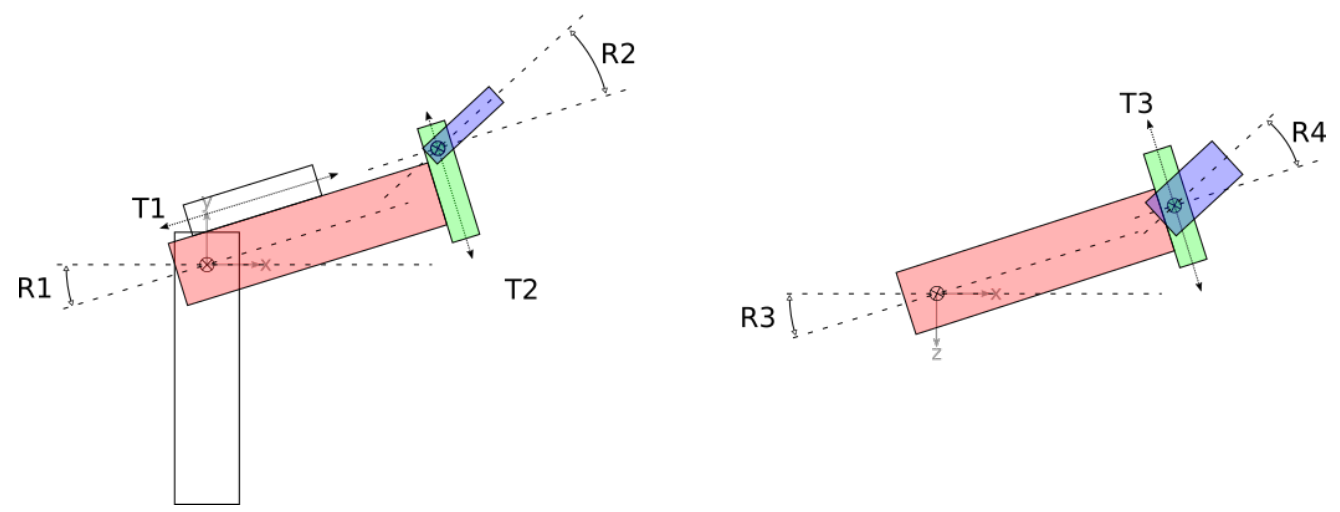

Figure 1: In this figure, a kinematic sketch of the seven degrees of freedom is shown. Rotations are designated R\# while translations are designated T\#.

After deciding on the necessary motions, a CAD-model was constructed to determine what parts were necessary. The CAD-model can be seen in Figure 2.

The mounts consist of the primary components found in Table 2 with a number of Bosch Rexroth profiles cut to desired lengths and shapes.

And lastly, in Figure 3 photos of the final mount is shown. 


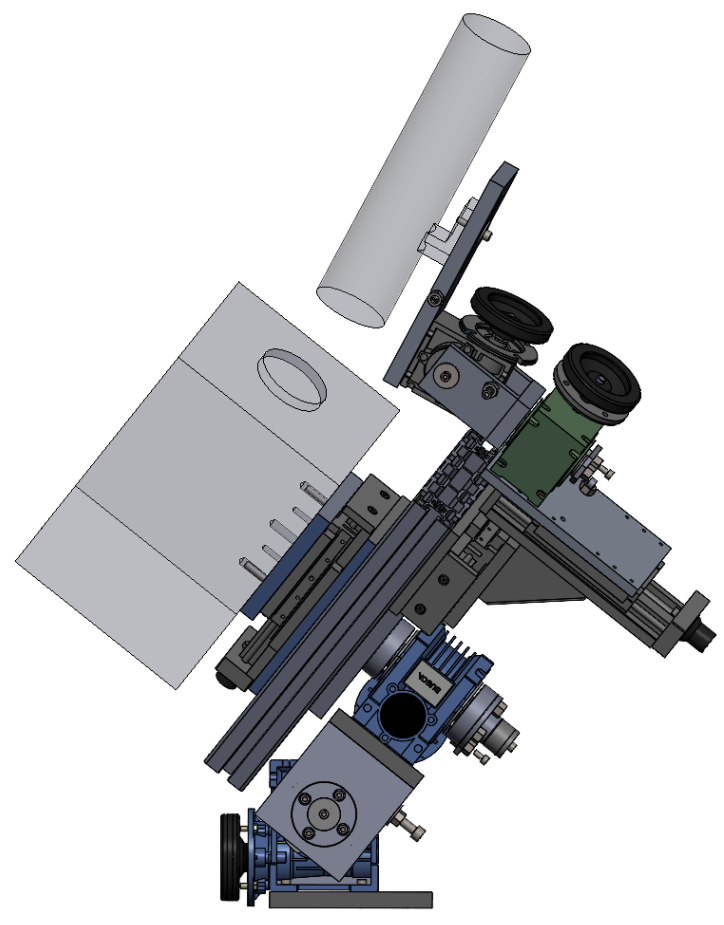

(a) Side view

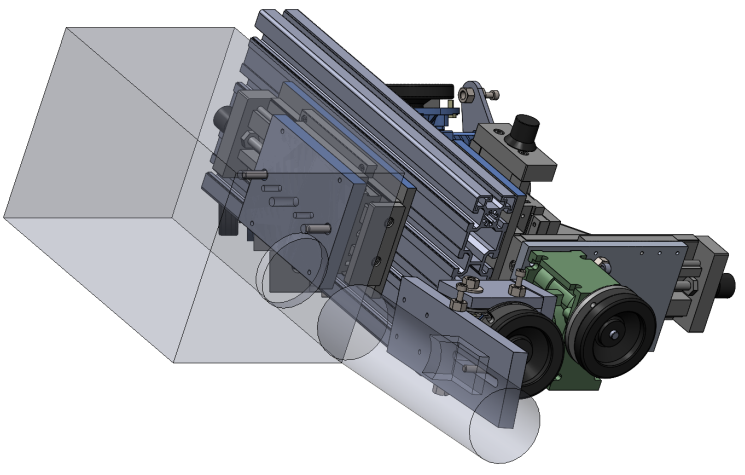

(b) Top view

Figure 2: (a) shows a CAD-model of the camera mount in side view, while (b) shows the mount in a topdown view.

Table 2: Components of each DoF.

\begin{tabular}{l|l|l|l|l} 
DoF & Type & Brand & Product no & Gear ratio \\
\hline R1 & Worm gear & BUSCK & SB040 & $1: 100$ \\
R2 & Worm gear & BUSCK & SB025 & $1: 60$ \\
R3 & Worm gear & BUSCK & SB040 & $1: 100$ \\
R4 & Worm gear & BUSCK & SB030 & $1: 80$ \\
T1 & Linear translation & Elesa-Ganter & GN 900-80-195-75-D-1 & N/A \\
T2 & Linear translation & Elesa-Ganter & GN 900-80-195-75-D-1 & N/A \\
T3 & Linear translation & Elesa-Ganter & GN 900-80-195-75-D-1 & N/A
\end{tabular}

\section{Performance}

To quantify the range of motion and accuracy that this construction allows for, measurements have been done using a laser pointer mounted at the tip of the lens mounting point. The movement of the laser along a wall is measured for different inputs. Each degree of freedom is subjected to one, two and three full rotations on the input axle making sure to always end with tension in the positive direction of rotation to minimize the effect of backlash. The range of motion is determined by turning or translating the relevant degree of freedom as far as the construction allows in both directions. The found values are presented in Table 3

Initial tests show that it is indeed possible to achieve focus across the sensor for combinations of rotations.

\section{Potential applications}

Anywhere the image plane and object plane are not aligned, but desired to be, the Scheimpflug angle can be applied. The general concept has near-limitless applications, but this specific mount allows solving the 


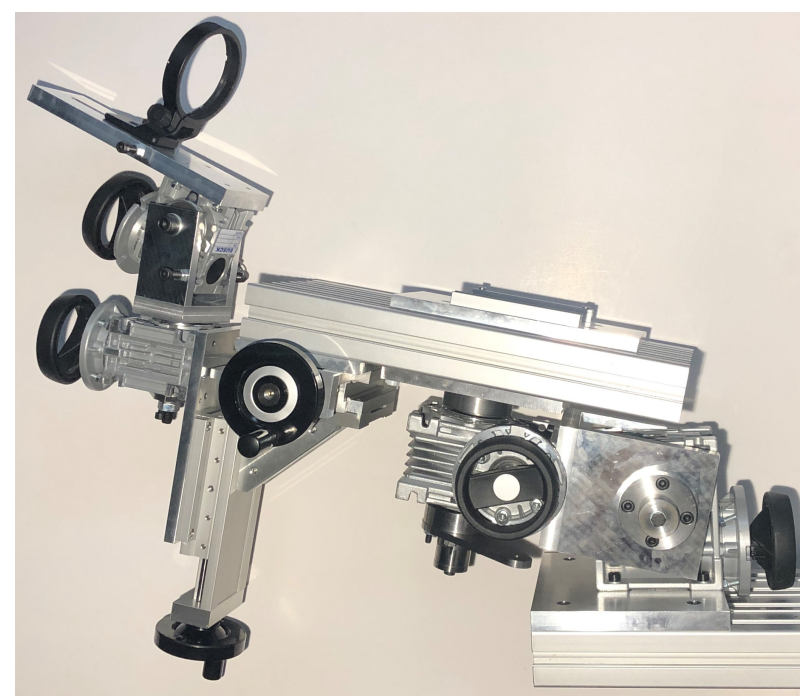

(a) Side view

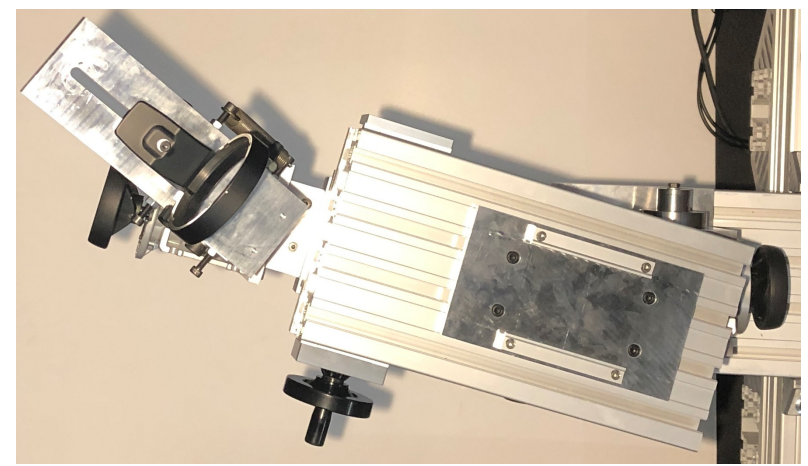

(b) Top view

Figure 3: (a) shows the actual camera mount in side view, while (b) shows the mount in a top-down view.

Table 3: Performance metrics.

\begin{tabular}{l|c|c} 
DoF & Increment per revolution & Range \\
\hline R1 & $3.6^{\circ}$ & {$\left[0^{\circ} ;>90^{\circ}\right]$} \\
R2 & $6.0^{\circ}$ & {$\left[-12.5^{\circ} ;>90^{\circ}\right]$} \\
R3 & $3.6^{\circ}$ & {$\left[<-90^{\circ} ;>90^{\circ}\right]$} \\
R4 & $4.5^{\circ}$ & {$\left[<-90^{\circ} ;>90^{\circ}\right]$} \\
T1 & $1.0 \mathrm{~mm}$ & {$[-32.5 \mathrm{~mm}-32.5 \mathrm{~mm}]$} \\
T2 & $1.0 \mathrm{~mm}$ & {$[-32.5 \mathrm{~mm}-32.5 \mathrm{~mm}]$} \\
T3 & $1.0 \mathrm{~mm}$ & {$[-32.5 \mathrm{~mm}-32.5 \mathrm{~mm}]$}
\end{tabular}

Scheimpflug focusing problem when using heavy equipment, both for the camera and the optics. This is especially relevant for optical measurement techniques, where high magnification and high temporal resolution is needed, as both increase the bulkiness of the equipment used.

Specifically, this setup allows the solution of the pseudo biaxial Scheimpflug focusing mentioned previously, when geometric or practical limitations hinder the necessary freedom needed to reduce the focusing to a monoaxial problem.

Examples of situations in which this extra freedom of positioning can come in handy could be:

a) Stereoscopic PIV when optical access to the plane of interest is limited. For example, imagine a wind tunnel in which the only viable camera position are on top, with optical access through a window, such as show in Figure 4(a). In this case, even a single camera would need to correct for the misalignment of image and object planes. However, when going to multiple cameras, the solution to the Scheimpflug focusing is less trivial, and with bulky equipment traditional mounts simply do not suffice. Here, our design would allow the decomposition of the ideal rotation into two separate rotations.

b) A pyramidal camera configuration for tomographic methods in which side-centered placement of the cameras is not possible, so they must be placed on the corners of the pyramid. This was the initial design for our setup, as seen in Figure 4(b).

\section{Final considerations}

The notion of a 2-axes Scheimpflug focusing has been reflected upon and the conclusion is that such a thing does not exist. At best, it is possible to talk about a pseudo biaxial Scheimpflug focusing, which occurs when it is not readily possible to place camera and lens in a manner simplifying the rotation to a single axis. This is essentially just an decomposition of rotations using Euler Angles. To allow for this niche case of Scheimpflug focusing, a camera mount that can carry both a heavy camera and a heavy optical 


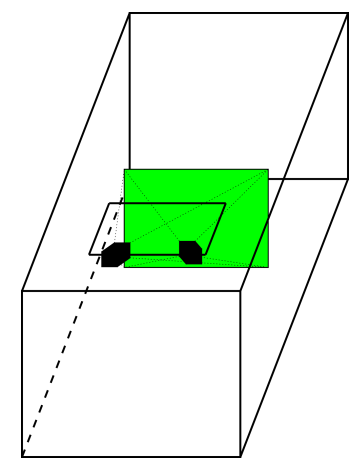

(a) Case a
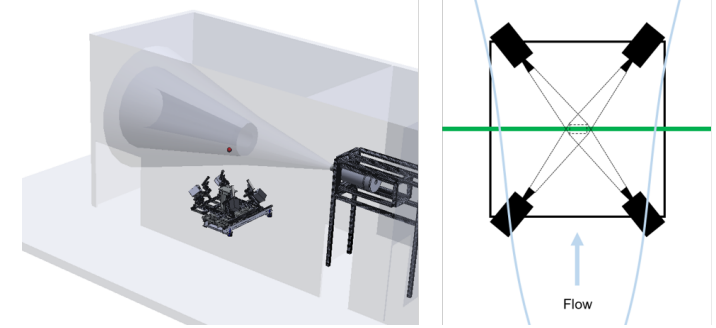

(b) Case b

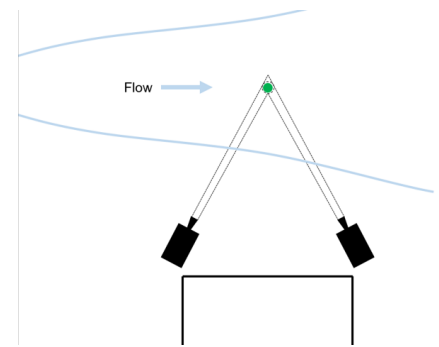

Figure 4: An example of stereoscopic PIV with limited optical access.

system has been designed, which allows independent control of the rotation of both camera and lens about their respective pitch and yaw axes. This is primarily useful when using high-speed equipment with highmagnification optical solutions in out-of-the-ordinary configurations. The mounts can, however, also easily handle monoaxial Scheimpflug focusing, which could prove useful when using heavy and bulky equipment in simpler setups, such as our current side-centered pyramidal camera configuration. The range of motion and rough estimates on sensitivity have been determined and presented, the range typically offering way more range of motion than what would feasibly produce a useful image on the sensor due to vignetting and similar effects.

\section{Acknowledgements}

This project has received funding from the European Research Council (ERC) under the European Unions Horizon 2020 research and innovation program (grant agreement No 803419). Furthermore, the author would like to acknowledge and thank Dr. Willert and his group at DLR Köln for their generous hosting and help.

\section{References}

Adrian RJ and Westerweel J (2011) Particle image velocimetry. Cambridge University Press

Raffel M, Willert CE, Scarano F, Kähler CJ, Wereley ST, and Kompenhans J (2018) Particle Image Velocimetry - A Practical Guide. Springer. $3^{\text {rd }}$ edition

Scheimpflug T (1904) Improved Method and Apparatus for the Systematic Alteration or Distortion of Plane Pictures and Images by Means of Lenses and Mirrors for Photography and for other purposes. Patent application. Great Britain Patent number 1196

Walker S (2001) Two-axes Scheimpflug focusing for particle image velocimetry. Meas. Sci. Technol. 13:112 